\title{
Overview of IEEE802.15.4g OFDM and its Applicability to Smart Building Applications
}

\author{
Jonathan Muñoz ${ }^{1,2}$, Emmanuel Riou ${ }^{2}$, Xavier Vilajosana ${ }^{3}$, Paul Muhlethaler ${ }^{1}$, Thomas Watteyne ${ }^{1}$ \\ 1 Inria, Paris, France. \\ \{jonathan.munoz, paul.muhlethaler, thomas.watteyne\} @inria.fr \\ 2 Gridbee Communications, Grasse, France. \\ emmanuel.riou@gridbeecom. com \\ ${ }^{3}$ Universitat Oberta de Catalunya, Barcelona, Catalonia, Spain. \\ xvilajosana@uoc.edu
}

\begin{abstract}
This paper compares the performance of two IEEE802.15.4 physical layers in the Smart Building context: 2.4 GHz O-QPSK and sub-GHz OFDM. The former has been in the IEEE802.15.4 standard since 2003, the latter was rolled into its 2015 revision. OFDM promises exceptional performance, in particular in environments with high external interference and multi-path fading. This paper starts with a comprehensive overview of IEEE802.15.4 and IEEE802.15.4g, with a particular focus on OFDM, its design drivers and modes of operation. The second half of this paper presents results from an exhaustive benchmarking campaign of both technologies in a building environment, and discusses lessons learnt. We show how OFDM has a higher range, even at $400 \mathrm{kbps}$ and $800 \mathrm{kbps}$ data rates. We then quantify the importance of frequency repetition in OFDM, and of using a wide communication channel, and we show how the use of OFDM can result in a 2-4 $\times$ decrease in power consumption compared to $2.4 \mathrm{GHz}$ O-QPSK. We conclude by recommending the use of OFDM option 1, with MCS2 for short $(<128 \mathrm{~B})$ frames, and MCS3 otherwise.
\end{abstract}

\section{INTRODUCTION}

Low-power wireless mesh networks encompass industrial [1], home [2], urban [3] and smart building [4] applications. In Smart Building applications [4], low-power wireless mesh networks are used to monitor and automate intrusion detection, fire detection, elevator monitoring, HVAC, and lighting, among others.

The IEEE802.15.4 standard has ruled the Smart Building application space for low-power wireless mesh solutions. Standards have been developed over the last decade to define an entire protocol stack, industrial alliances have built around them, and countless companies, big and small, sell IEEE802.15.4-based Smart Building solutions.

These solutions mostly use the $2.4 \mathrm{GHz}$ version of IEEE802.15.4, with O-QPSK modulation, DSSS, and $127 \mathrm{~B}$ frames. This physical layer provides a trade-off between energy consumption, communication range, and reliability which is absolutely suited to Smart Building applications. Tens of thousands of networks based on that physical layer are operating today, and achieve over $99.999 \%$ end-to-end reliability and over a decade of battery lifetime. It is not the goal of this paper to argue against IEEE802.15.4.

Yet, there are a number of recent developments in the physical layers of the IEEE802.15.4 standard. Its 2015 revision

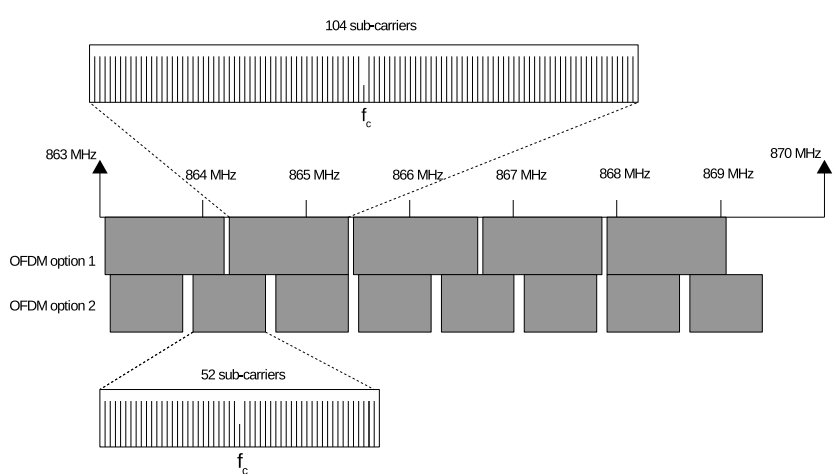

Fig. 1: In OFDM, data is transmitted on multiple sub-carriers. Multiple sub-carriers are grouped into channels; the OFDM option determines how this grouping is done.

includes the IEEE802.15.4g amendment, which defines new physical layers. These have been designed for smart utility applications, and focus on range. Among these is OFDM (Orthogonal Frequency Division Multiplexing), a physical layer which has been extensively used in high-end wireless systems, and which is now entering the low-power wireless space. On paper, OFDM offers longer range, higher bandwidth and better handling of external interference and multi-path fading. This paper explores these claims, experimentally, in the Smart Building application space.

The remainder of this paper is organized as follows. Section II gives an overview of IEEE802.15.4 and IEEE802.15.4g, and presents related work. Section III lists the contributions of this paper. Section IV details the experimental setup. Section V discusses the experimental results and what lessons can be learnt from them. Section VI concludes this paper.

\section{OVERVIEW AND RELATED WORK}

This section provides a complete overview of both IEEE802.15.4 (Section II-A) and IEEE802.15.4g (Section II-B). Both sections give a comprehensive overview of the technology and go over previously-published related work. 


\section{A. IEEE802.15.4 2.4 GHz}

The IEEE802.15.4 standard [5] has been almost synonymous to smart building applications. This standard defines the physical layer (modulation, data rate, transmit power). All major chip vendors have cheap ${ }^{1}$ IEEE802.15.4-compliant radio chips in their catalog, making it a very accessible technology. The communication range with those chips (assuming a $0 \mathrm{dBm}$ transmit power and a sensitivity around $100 \mathrm{dBm}$ ) is in the order of $100-200 \mathrm{~m}$ outdoors and 20$50 \mathrm{~m}$ indoors. Their power consumption is $5-25 \mathrm{~mA}$ at $3.6 \mathrm{~V}$, making it possible to achieve a decade of battery lifetime when duty cycling. Because it is unlicensed and world-wide, the $2.4 \mathrm{GHz}$ frequency band is the most used for smart building applications.

At the physical layer, IEEE802.15.4 uses O-QPSK modulation with DSSS. It cuts the $2.400-2.485 \mathrm{GHz}$ frequency bands into 16 orthogonal frequencies, separated by $5 \mathrm{MHz}$, each $2 \mathrm{MHz}$ wide. The maximum frame size of IEEE802.15.4 is $127 \mathrm{~B}$; the data rate is $250 \mathrm{kbps}$. This means that it takes an IEEE802.15.4 radio roughly $4 \mathrm{~ms}$ to transmit a $127 \mathrm{~B}$ frame.

The first version of IEEE802.15.4 was ratified in 2003. In the decade that followed, several fully standards-compliant protocol stacks were built on top of the IEEE802.15.4 physical layer. The protocol stack mainly defines how these radios are duty cycled to conserve energy (at the "Medium Access Control" - MAC - layer), and how to organize a network deployed throughout a building as a multi-hop mesh (at the routing layer). Countless proposals of MAC and routing combinations have been proposed by academia; a couple such as the RPL routing protocol [6] - have made it through the standardization process.

Industrial alliances have formed, which typically put together several standards to form a complete protocol stack, and serve as labeling/certification bodies. The most prevalent examples in the Smart Building space are ZigBee ${ }^{2}$ and Thread ${ }^{3}$.

Tens of thousands of low-power wireless mesh networks are operating today, using variants of these protocols and standards. A large number of companies are working in the Smart Buildings space. One example is Assa Abloy ${ }^{4}$, which commercializes wireless door opening solutions. Another example is Yanzi Networks ${ }^{5}$, which commercializes indoor temperature, humidity and presence sensors, as well as controllable power sockets.

There are two main challenges for making a network composed of IEEE802.15.4 devices, operating at $2.4 \mathrm{GHz}$, reliable.

The first challenge is external interference. At $2.4 \mathrm{GHz}$, external interference mainly comes from IEEE802.11 (WiFi) and IEEE802.15.1 (Bluetooth).

Khaleel et al. [7] investigate the cross-interference between IEEE802.15.4 and IEEE802.11b (WiFi). They use a Cross-

\footnotetext{
${ }^{1}$ Sub-5 USD IEEE802.15.4 radio chips are commonplace.

$2 \mathrm{http}: / /$ www.zigbee.org/

3 https://threadgroup.org/

${ }^{4}$ https://www.assaabloy.com/

${ }^{5}$ https://www.yanzi.se/
}

bow Telos device equipped with an IEEE802.15.4-compliant CC2420 radio to sense the frequency spectrum by using RSSI, under different $\mathrm{WiFi}$ data rate conditions. They show that when there is a WiFi connection of $3 \mathrm{Mbit} / \mathrm{s}$, the probability of failure to access the medium for a IEEE802.15.4 device reaches $90 \%$, when operating on the same frequency as the WiFi connection.

Watteyne et al. [8] conduct an experiment to record the connectivity between 350 nodes in a typical office environment, using the IoT-lab large-scale testbed [9]. These nodes communicate on each of the 16 available frequencies at $2.4 \mathrm{GHz}$. They show the impact of WiFi interference on the reliability of the IEEE802.15.4 wireless links: even when the WiFi network sits idle, IEEE802.11 beaconing causes a significant number of links to drop from 90\% to 70-80\% Packet Delivery Ratio (PDR).

The second challenge is multi-path fading. In any indoor environment, objects in the surroundings of a wireless link cause a reflection of the radio signal. These different "echoes" of the same signal reach the receiver's antenna at slightly different times. All these reflections can interfere constructively, increasing the signal strength. Yet, they can also interfere destructively, making the communication between transmitter and receiver impossible.

Watteyne et al. [10] visualize the effect of multi-path fading. They install a transmitting node on a robotic arm which moves inside a $20 \mathrm{~cm}$ by $35 \mathrm{~cm}$ plane, with a $1 \mathrm{~cm}$ step, yielding 735 positions. At each position, the transmitting node sends 100029 byte frames to the receiver node $1 \mathrm{~m}$ away. The experiment is repeated over each of the 16 available frequencies. Results show that the PDR of the wireless link between transmitter and receiver can swing from $100 \%$ to $0 \%$ by moving the transmitter by just $3 \mathrm{~cm}$. This is entirely due to multi-path fading.

New techniques have appeared to cope with external interference and multi-path fading in IEEE802.15.4 networks operating at $2.4 \mathrm{GHz}$. The most disruptive is arguably Time Slotted Channel Hopping (TSCH), a MAC approach by which tightly synchronized nodes, heavily duty cycle their radio to conserve energy, and use frequency hopping to combat interference and multi-path fading. TSCH has been the default MAC approach in IEEE802.15.4, since its 2015 revision. Further standardization at the IETF 6TiSCH working group defines how to combine TSCH with IPv6. The authors in [11] present the performance of SmartMesh IP, a commercial TSCH solution, which yields over $99.999 \%$ end-to-end reliability, and over a decade of battery lifetime. TSCH (and 6TiSCH) networks are widely regarded as the future for low-power wireless networks, and are the base for all major open source implementations [12] as well as several commercial ongoing implementations.

Clearly, using 6TiSCH can yield wire-like reliability and a decade of battery lifetime from an IEEE802.15.4 network operating at $2.4 \mathrm{GHz}$. This paper looks at whether using a different physical layer - possibly combined with $6 \mathrm{TiSCH}-$ has the potential to yield even better performance. A whole 
new set of sub-GHz physical layers has been developed within the IEEE802.15.4g task group. The goal of this paper is to explore their potential.

\section{B. IEEE802.15.4g sub-GHz}

The IEEE802.15.4g amendment [13] was created for Smart Utility Network (SUN) applications. One strong requirement, to be able to build neighborhood-wide (mesh) networks, is a multi-km range. The IEEE802.15.4g amendment, first published in 2012, was rolled into the main IEEE802.15.4 specification in its 2015 revision [14] $]^{6}$.

IEEE 802.15.4g introduces three alternative physical layers (PHYs): FSK (Frequency Shift Keying), O-QPSK (OffsetQuadrature Phase Shift Keying) and OFDM (Orthogonal Frequency Division Multiplexing). Each physical layer was designed for a specific market segment, and is marketed as having distinct advantages. FSK increases the transmit power efficiency by the constant envelope of the signal. O-QPSK shares the characteristics of IEEE802.15.4 DSSS O-QPSK. OFDM provides high data rates, and is designed to operate in environments with frequency selective fading, such as indoors [14]. In all cases, compliant radio chips can exchange frames of up to $2047 \mathrm{~B}$.

FSK and O-QPSK are conventional well-known modulation techniques. While OFDM is commonly used in advanced systems, its introduction to low power wireless devices is new. OFDM was created to combat multi-path fading. Like TSCH, it exploits frequency diversity. But while TSCH does so at the MAC layer, OFDM does so directly at the physical layer. In OFDM, a frequency band (called "channel") is divided into numerous frequencies ("sub-carriers"). The sub-carriers are far enough apart in frequency to be orthogonal: they do not interfere with one another. An OFDM symbol is the combination of the sub-carriers, each carrying a portion of the information to be transmitted. Each sub-carrier is modulated with a low-order modulation (BPSK, O-QPSK or 16-QAM). Combining the sub-carriers is equivalent to having a high order signal modulation (e.g., 16,777,216-PSK for 24 bits per symbol), making it possible to achieve high data rates. Fig. 1 shows the OFDM channels in IEEE802.15.4g $\mathrm{g}^{7}$, where communication uses multiple sub-carriers, separated by a constant equal frequency distance $\Delta f$. In the center frequency $f_{c}$, no information is sent (null tone).

In OFDM, the effective duration of a symbol $\left(t_{s}\right)$ is $96 \mu$ s. To ensure orthogonality between sub-carriers, they are separated by $1 / t_{s}$, or $10,416.667 \mathrm{~Hz}$. The standard describes 4 ways of grouping sub-carriers to form an OFDM symbol; they are called "options", and are numbered from 1 to 4 . Table I shows the number of sub-carriers for each option, as well as the bandwidth it occupies.

\footnotetext{
${ }^{6}$ Strictly speaking, IEEE802.15.4g was rolled into IEEE802.15.4 in 2015. It is however common to (still) refer to this part of the IEEE802.15.4 specification as "IEEE802.15.4g", as we do throughout this paper.

${ }^{7}$ Throughout this paper, we use the European $863-870 \mathrm{MHz}$ frequency band Our results hold for other frequency bands, including the $902-928 \mathrm{MHz}$ US band, the only difference being the number of available channels.
}

\begin{tabular}{|l|r|r|r|r|}
\hline & \multicolumn{1}{|c|}{ option } & \multicolumn{1}{c|}{ option } & \multicolumn{1}{c|}{ option } & option \\
& 1 & \multicolumn{1}{c|}{2} & \multicolumn{1}{c|}{3} & \multicolumn{1}{c|}{4} \\
\hline \# sub-carriers & 104 & 52 & 26 & 14 \\
(data/pilot) & $96 / 8$ & $48 / 4$ & $24 / 2$ & $12 / 2$ \\
\hline Channel width $(\mathrm{kHz})$ & 1094 & 552 & 281 & 156 \\
\hline Space between channels (kHz) & 1200 & 800 & 400 & 200 \\
\hline \# Channels & 5 & 8 & 17 & 34 \\
\hline
\end{tabular}

TABLE I: The OFDM option specifies how many sub-carriers are used in one channel.

\begin{tabular}{|l|c|c|c|c|}
\hline & $\begin{array}{c}\text { option } \\
1\end{array}$ & $\begin{array}{c}\text { option } \\
2\end{array}$ & $\begin{array}{c}\text { option } \\
3\end{array}$ & $\begin{array}{c}\text { option } \\
4\end{array}$ \\
\hline $\begin{array}{l}\text { MCS0 } \\
\text { BPSK rate 1/2 } \\
4 \times \text { freq. rep. }\end{array}$ & $100 \mathrm{kbps}$ & $50 \mathrm{kbps}$ & - & - \\
\hline $\begin{array}{l}\text { MCS1 } \\
\text { BPSK rate 1/2 } \\
2 \times \text { freq. rep. }\end{array}$ & $200 \mathrm{kbps}$ & $100 \mathrm{kbps}$ & $50 \mathrm{kbps}$ & - \\
\hline $\begin{array}{l}\text { MCS2 } \\
\text { QPSK rate 1/2 } \\
2 \times \text { freq. rep. }\end{array}$ & $400 \mathrm{kbps}$ & $200 \mathrm{kbps}$ & $100 \mathrm{kbps}$ & $50 \mathrm{kbps}$ \\
\hline $\begin{array}{l}\text { MCS3 } \\
\text { QPSK rate 1/2 } \\
\text { no freq. rep. }\end{array}$ & $800 \mathrm{kbps}$ & $400 \mathrm{kbps}$ & $200 \mathrm{kbps}$ & $100 \mathrm{kbps}$ \\
\hline $\begin{array}{l}\text { MCS4 } \\
\text { QPSK rate 3/4 } \\
\text { no freq. rep. }\end{array}$ & - & $600 \mathrm{kbps}$ & $300 \mathrm{kbps}$ & $150 \mathrm{kbps}$ \\
\hline $\begin{array}{l}\text { MCS5 } \\
\text { 16-QAM rate 1/2 } \\
\text { no freq. rep. }\end{array}$ & - & $800 \mathrm{kbps}$ & $400 \mathrm{kbps}$ & $200 \mathrm{kbps}$ \\
\hline $\begin{array}{l}\text { MCS6 } \\
\text { 16-QAM rate 3/4 } \\
\text { no freq. rep. }\end{array}$ & - & - & $600 \mathrm{kbps}$ & $300 \mathrm{kbps}$ \\
\hline
\end{tabular}

TABLE II: The MCS setting specifies the modulation used on each sub-carrier, its symbol rate, and whether frequency repetition is used. Combined with an OFDM option, this yields a data rate. A dash ('-') indicates a combination that does not exist. Colored cells are settings explored in Section IV.

The sub-carrier modulation is specified by the Modulation and Coding Scheme (MCS). There are 7, numbered from 0 to 6 . The data rate of the signal is given by the combination of the OFDM option and MCS. Table II shows the details of the MCS and the data rates for each OFDM option/MCS combination.

Frequency repetition is an OFDM technique in which more than one sub-carrier transports the same information. While it reduces the effective data rate of the transmission, it makes the signal much more robust against multi-path fading. That is, even if one sub-carrier is not successfully demodulated, there is another sub-carrier (at a different frequency) that carries the same data.

Any IEEE802.15.4g-compliant chip must implement a physical layer with 2-FSK modulation and $50 \mathrm{kbps}$ data rate. All other physical layers are optional. Each physical layer allows further parametrization (data rate, bandwidth). The result is that IEEE802.15.4g comprises an astounding 31 different physical layer options, with data rates ranging from $6.25 \mathrm{kbps}$ to $800 \mathrm{kbps}$.

With such a variety of settings, it is hard for an implementor to understand which setting to use. This paper aims 
to contribute to providing an answer. We start by looking at already-published related work, with a particular focus on hands-on performance evaluation in specific scenarios, ideally comparing the different physical layers. We then conduct our own experiments to compare sub-GHz OFDM with the traditional $2.4 \mathrm{GHz}$ O-QPSK to see whether, as an end user, there is some advantage. We are particularly interested in the higher data rates modes of OFDM, and in the impact of frequency repetition on reliability.

We have conducted the experiments introduced in Section IV on all IEEE802.15.4g modes. For reasons of space ${ }^{8}$, we only present the modes which give the reader the most insights: option 1 MCS2 (400 kbps, $1094 \mathrm{kHz}$ bandwidth, $2 \times$ frequency repetition), option $1 \mathrm{MCS} 3$ (800 kbps, $1094 \mathrm{kHz}$ bandwidth, no frequency repetition), option 2 MCS5 (800 kbps, $552 \mathrm{kHz}$ bandwidth, no frequency repetition).

This paper is obviously not the first to look at OFDM in a low-power wireless context. Several teams have worked on building complete IEEE802.15.4g-based solutions. That is, given a physical layer, assemble a protocol stack with existing standards, and evaluating the resulting network in some pilot deployment.

Dias et al. [15] evaluate a protocol stack they designed for smart metering in an industrial environment. The solution is based on IEEE802.15.4g, combined with an upper stack developed by the IETF (6LoWPAN, RPL OF1, TLS-DTLS and DLMS/COSEM). They use AT86RF215 chips configured to communicate at $915 \mathrm{MHz}$ using O-QPSK modulation and $250 \mathrm{kbps}$ data rate. They test a 10-node network deployed for 20 days in a $120 \mathrm{~m} \times 40 \mathrm{~m}$ warehouse. The nodes form a multi-hop network around a border router located in the corner of the building. Using simple reachability tests, they show that packet loss ratio increases rapidly with the number of hops, with almost $90 \%$ loss at $4-5$ hops. The round-trip time increases by approximately $15 \mathrm{~ms}$ per hop.

Mochizuki et al. [16] implement a Wireless Smart Utility Network (Wi-SUN) system. They propose up-links transmissions ("UP", toward the Border Router) at $20 \mathrm{~mW}(+13 \mathrm{dBm})$, and down-link transmissions ("DL", toward the nodes) at $250 \mathrm{~mW}(+24 \mathrm{dBm})$. The deployment is performed in the city of Kyoto, Japan, consisting of a Border Router and a mobile Measurement Station. The Border Router is located on the roof of the Kyoto City Hall, $17 \mathrm{~m}$ high. Data rate is set to $100 \mathrm{kbps}$, modulation to $2-\mathrm{GFSK}$, frame length to $250 \mathrm{~B}$, and frames are separated by $50 \mathrm{~ms}$. The Measurement Station is placed at several distances from the Border Router, in both Light-of-Sight (LOS) and Non-Light-of-Sight (NLOS) conditions. 1000 frames are transmitted from the Border Router to the Measurement Station, for each of its locations. The authors record the PDR for different positions of the Measurement Station. They show that, in LOS conditions, a higher transmission power at the Border Router provides more stable communication. By changing the transmit power of the

\footnotetext{
${ }^{8}$ The complete results can be found in a technical report, companion to this paper.
}

base station from $20 \mathrm{~mW}$ to $250 \mathrm{~mW}$, its communication range increases from $150 \mathrm{~m}$ to $650 \mathrm{~m}$.

The related work reviewed so far focuses on building entire systems. None of the publications pays particular attention to the IEEE802.15.4g setting (modulation, data rate) used. We believe is that OFDM is very applicable for the Smart Building space, and we use this paper to argue why. We are convinced that OFDM has not received sufficient attention for two reasons. First, IEEE802.15.4-OFDM is a recent technology. The Atmel AT86RF215 ${ }^{9}$, arguably the most used radio capable of OFDM, was only available since Q3 $2015^{10}$. Second, OFDM is perceived as being very energy hungry and complex [17], and therefore not applicable for battery-powered low-power wireless mesh solutions.

What is missing is work that evaluates IEEE802.15.4g OFDM experimentally, and assesses its suitability for lowpower wireless mesh-based Smart Building solutions. Lee et al. [18] are, to the best of our knowledge, the only authors who have done a similar study, on a home-made implementation of IEEE802.15.4g OFDM (combining a 32-bit micro-controller, an FPGA and a radio frequency ASIC). Their article is, however, focused on the feasibility of a smart home utility service using IEEE802.15.4g FSK and OFDM. They build a data concentrator and remote monitor connected to the Internet and then check the real status of the water and electricity consumption. No details are given about the location of the nodes and the distance covered by the radio links.

Our paper contributes to this missing body of work. Section III details our goal and lists our contributions.

\section{CONTRIBUTIONS}

This study compares sub-GHz IEEE802.15.4g-OFDM options 1 and 2 in their higher data rates with IEEE802.15.4 O-QPSK PHY at $2.4 \mathrm{GHz}$, from the user's point of view, in smart building applications.

The contribution of this study is five-fold:

1) We show that the Packet Delivery Ratio (PDR) of IEEE802.15.4g-OFDM is higher than for IEEE802.15.4 O-QPSK, for all locations.

2) We show that this holds even when sending $2047 \mathrm{~B}$ frames over IEEE802.15.4g-OFDM and 127 B over IEEE802.15.4 O-QPSK.

3) We show how frequency repetition can double the PDR of marginal links.

4) We show the importance of using wide channels.

5) We further show that the charge consumed for sending a $127 \mathrm{~B}$ packet over IEEE802.15.4g-OFDM is at least 2 times lower than IEEE802.15.4 O-QPSK.

We understand the impact changing frequencies $(2.4 \mathrm{GHz}$ and sub-GHz) has on many different metrics, including range. Some might even qualify comparing $2.4 \mathrm{GHz}$ O-QPSK with sub-GHz OFDM as "unfair". Yet, we approach this comparison purely from an end-user point of view: given the choice

$$
9
$$

http://ww1.microchip.com/downloads/en/DeviceDoc/ Atmel-42415-WIRELESS-AT86RF215_Datasheet.pdf

10 http://www.atmel.com/about/news/release.aspx?reference=tcm:26-64181 
between technologies, and given that all operate in unlicensed bands, does it make sense to use Sub-GHz OFDM for a Smart Building application?

\section{EXPERIMENTAL SETUP}

We conduct this study through a number of experiments. To run an experiment, we use 4 nodes: one is a transmitter (TX), the other 3 are receivers (RX). During an experiment, the nodes loop through all combinations of modulation, frequency and frame length, and, for each, measure the PDR of the link.

This section provides enough details for the reader to be able to replicate the tests and results. We describe the hardware (Section IV-B), software (Section IV-C), radio characteristics (Section IV-D), and deployments (Section IV-E).

\section{A. Foreword: Alias and Color Codes}

We want to compare the performance of $2.4 \mathrm{GHz}$ IEEE802.15.4 O-QPSK and sub-GHz IEEE802.15.4g OFDM. The former is almost synonymous with smart building applications, and therefore represents a baseline. The latter is newer, and promises exceptional performance in environment in which multi-path fading is very present. Our test considers all 31 radio settings, covering all IEEE802.15.4 and IEEE802.15.4g modulations. For reasons of space, and to provide the reader with the most insightful information, we only present results for the following 4 PHYs: $2.4 \mathrm{GHz} O$ QPSK at $250 \mathrm{kbps}$, sub-GHz OFDM option 1 at $400 \mathrm{kbps}$, subGHz OFDM option 1 at $800 \mathrm{kbps}$ and sub-GHz OFDM option 2 at $800 \mathrm{kbps}$. Table III provides an "alias" and assigns a color for each PHY. These will be used throughout the remainder of this paper.

\section{B. Hardware}

Each node is equipped with an Atmel AT86RF215 radio chip, which implements both IEEE802.15.4 (2.4 GHz) and IEEE802.15.4g (sub-GHz). We use the AT86RF215 Xplained Pro reference board - manufactured by Atmel - to ensure the setup follows the chip vendor's recommendations. This board has two SMA connectors on which we connect two $2 \mathrm{dBi}$ omni-directional antennas, one for $2.4 \mathrm{GHz}$, one for sub-GHz.

A node (depicted in Fig. 2) consists of a Raspberry Pi 3 (rPi) model B connected to the radio board over an SPI bus. The electronics and connectors are housed in a plastic box, with the two antennas sticking out. The box is self-contained and powered by a 22,000 mAh battery bank, more than enough charge to power the node during an experiment.

\section{Software}

The $\mathrm{rPi}$ of each node runs a Linux Debian distribution, and is connected to the Internet over WiFi. We connect over $\mathrm{SSH}$ to each node to remotely launch the test scripts. The test scripts are written in Python, and drive the radio throughout an experiment ${ }^{11}$. The scripts are responsible for having the TX node loop through all combinations of modulation, frequency

\footnotetext{
11 As an online addition to this paper, all the software is published under an open-source license at https://github.com/openwsn-berkeley/range_test.
}

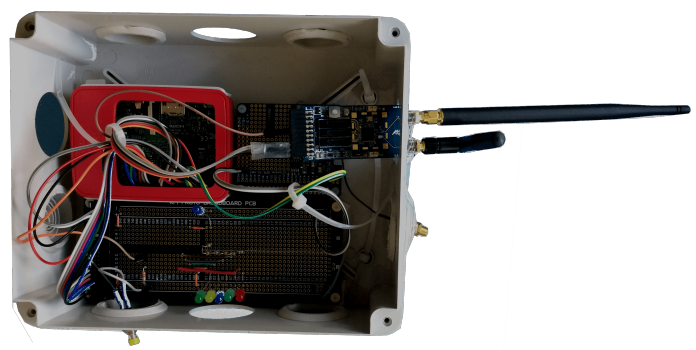

Fig. 2: Picture of a test node. Four such test nodes are used in the experiments, each equipped with an AT86RF215 radio communicating on both $2.4 \mathrm{GHz}$ IEEE802.15.4 O-QPSK and sub-GHz IEEE802.15.4g OFDM.

and frame length, and, for each, send 100 frames. On the RX side, the scripts are responsible for (re-)configuring the radio so it is listening on the same frequency using the same modulation as the TX node at the same time. TX and RX nodes are synchronized over NTP. Appropriate guard times are introduced to ensure that the RX node is listening when the TX node transmits a frame.

The frame lengths considered depend on the PHY. For OFDM, the TX node sends frames of lengths $127 \mathrm{~B}$ and 2047 B. For IEEE802.15.4 O-QPSK, the TX node sends frames of length $127 \mathrm{~B}$. Similarly, the frequencies considered depend on the physical layer. For OFDM, there are 5 and 8 available frequencies for option 1 and 2, respectively. For the $2.4 \mathrm{GHz}$ frequency band, there are 16 frequencies. In all cases, the inter-frame spacing is $20 \mathrm{~ms}$ and the TX power is $+8 \mathrm{dBm}$.

An experiment - looping through all modulations, frequencies and frame lengths - takes roughly 30 min. During an experiment, an RX node logs, for each frame received, the modulation and frequency it listens on, the counter contained in the frame, whether its FCS is correct and the RSSI value. Because 100 frames are sent for each modulation/frequency/length, the PDR for that setting can be computed.

\section{Radio Characteristics}

Table III gives the current draw of the AT86RF215 radio chip, at $3.3 \mathrm{~V}$, for each radio setting. Because the chip's datasheet does not provide the current draw for each setting, we measured it. For each radio setting, we configure the TX node to transmit in continuous transmission mode, and measure the current draw using an ammeter.

Table III also details the sensitivity of the AT86RF215 radio chip, for each radio setting, as read from the datasheet ${ }^{12}$.

\section{E. Deployments}

Fig. 3 shows a floorplan of the deployment area, the Inria office building in Paris, France. The ceiling is metallic, the floor is covered with carpet, external concrete walls have glass

12 The sensitivity for IEEE802.15.4g is defined as the RSSI which yields $10 \%$ PER with $250 \mathrm{~B}$ frames. The sensitivity for IEEE802.15.4 is defined as the RSSI which yields $1 \%$ PER with 20 B frames. 


\begin{tabular}{|c|c|c|c|c|c|c|c|c|}
\hline Radio Settings & Freque & & Modulation & MCS & Data Rate & TX current $(+8 \mathrm{dBm})$ & RX current & RX Sensitivity \\
\hline "O-QPSK" & $2.400-2.484$ & $\mathrm{GHz}$ & O-QPSK & & $250 \mathrm{kbps}$ & $64.5 \mathrm{~mA}$ & $32.4 \mathrm{~mA}$ & $-104 \mathrm{dBm}$ \\
\hline “OFDM1@400” & $863-870$ & $\mathrm{MHz}$ & OFDM option 1 & 2 & $400 \mathrm{kbps}$ & $70.0 \mathrm{~mA}$ & $30.5 \mathrm{~mA}$ & $-107 \mathrm{dBm}$ \\
\hline "OFDM1@800” & $863-870$ & $\mathrm{MHz}$ & OFDM option 1 & 3 & $800 \mathrm{kbps}$ & $70.3 \mathrm{~mA}$ & $30.5 \mathrm{~mA}$ & $-104 \mathrm{dBm}$ \\
\hline "OFDM2@800" & $863-870$ & $\mathrm{MHz}$ & OFDM option 2 & 5 & $800 \mathrm{kbps}$ & $70.8 \mathrm{~mA}$ & $31.0 \mathrm{~mA}$ & $-101 \mathrm{dBm}$ \\
\hline
\end{tabular}

TABLE III: The PHY characteristics evaluated in this paper.

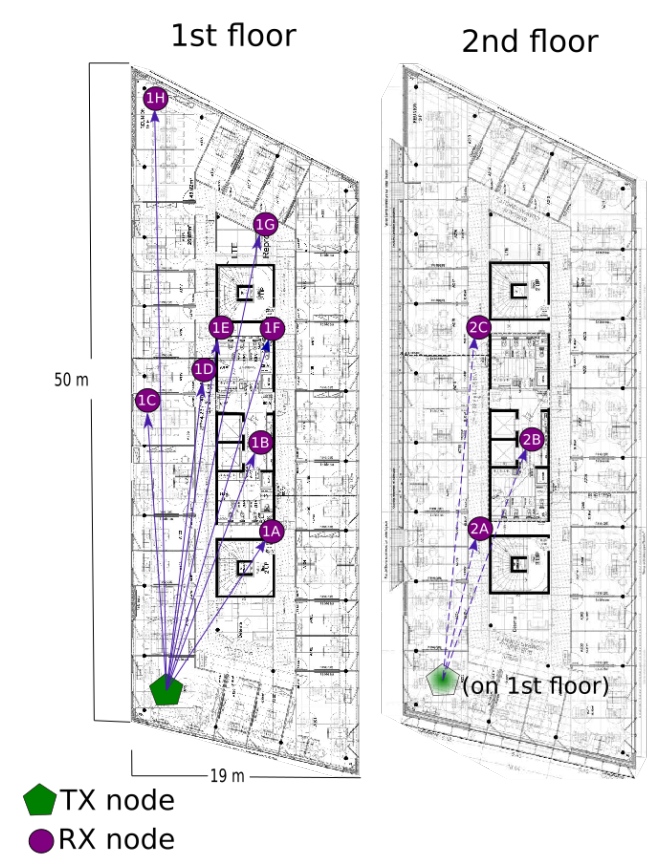

Fig. 3: Floorplan of the deployment area.

windows. Two concrete staircases and two elevator shafts are at the center of each floor.

A total of 3 experiments are conducted, during business hours (people are moving around and WiFi being actively used). All nodes are mounted on $1.8 \mathrm{~m}$ PVC poles. Between experiments, only the RX nodes are relocated, the TX node stays in the same position. Over the course of the 3 experiments, the RX nodes are placed at 8 locations on the same floor as the TX node, and at 3 locations on the floor above.

\section{EXPERIMENTAL RESULTS}

In total, we collected 57,200 atomic measurements, one for each frame, RX location, frequency, frame length, and index. This dataset contains a wealth of information. The goal of this section is to explore this dataset and extract the lessons they contain.

\section{A. The Longer Range of OFDM}

Table IV gives the average PDR value over all frequencies available per PHY and per RX node location. It shows three tiers of RX positions. In the first tier (positions 1A through $1 \mathrm{E})$, the receiver and transmitter nodes are close. PDR is over $80 \%$ in all cases, for both OFDM and O-QPSK. In the second tier $(1 \mathrm{~F}-1 \mathrm{H})$, the PDR of O-QPSK starts decreasing (down to $62 \%$ ), whereas the PDR of OFDM stays above $75 \%$. In the third tier (2A-2C), OFDM still offers some connectivity while O-QPSK is not able to get any frame across.

Fig. 4 depicts the PDR/RSSI relationship for nodes 1F, $1 \mathrm{G}$ and $1 \mathrm{H}$. Each dot corresponds to the PDR/average RSSI relationship for 100 frames of $127 \mathrm{~B}$ sent on one frequency ${ }^{13}$. All the OFDM samples exhibit a higher RSSI than O-QPSK. OFDM is well inside the sensitivity of its radio, O-QPSK presents samples closer to the sensitivity. This is expected given the difference in frequency [19].

Yet, there is more involved than simply the difference in frequency. Because it operates at $2.4 \mathrm{GHz}, \mathrm{O}-\mathrm{QPSK}$ should suffer from external interference from WiFi. By transmitting on multiple frequencies at the same time, OFDM should also be more robust against multi-path fading. We witness both phenomena in Sections V-B and V-C, respectively.

\section{B. The (Limited) Impact of WiFi Interference over O-QPSK}

Fig. 5 shows the average PDR for each IEEE802.15.4 frequency at $2.4 \mathrm{GHz}$, for positions $1 \mathrm{~F}, 1 \mathrm{G}$ and $1 \mathrm{H}$. At the same time as the experiment was conducted, WiFi was operating in the building on IEEE802.11 channels $1(2.412 \mathrm{GHz})$, $6(2.437 \mathrm{GHz})$ and $11(2.462 \mathrm{GHz})$. WiFi channels are $22 \mathrm{MHz}$ wide, each covering roughly 4 IEEE802.15.4 frequencies. Fig. 5 clearly shows a degradation in the PDR for IEEE802.15.4 frequencies in the WiFi channels, from 80-90\% down to $50-60 \%$. Yet, this impact is small, and will just require an IEEE802.15.4 radio to retransmit more often when operating in a WiFi channel. That effect alone does not explain the better PDR of OFDM presented in Table IV.

\section{The Power of Frequency Repetition}

Table IV shows that, at 127 B, OFDM1@400 and OFDM1@800 perform the same. The difference between OFDM1@400 and OFDM1@800 is that only the former uses a $2 \times$ frequency repetition, meaning that each portion of data is repeated on two different frequencies. This means that if multipath fading prevents the receiver from correctly decoding the frame on one frequency, it should be able to on the second copy. Of course, enabling $2 \times$ frequency repetition reduces the data rate by half.

When increasing the frame length, however, things change. With 2047 B frames, it takes the radio longer to transmit the frame. At a constant bit error rate, it is normal to have a higher frame error rate. Table IV clearly shows the benefit of frequency repetition: from RX location for example enabling frequency repetition for a 2047 B frame raises the PDR from $44 \%$ to $80 \%$.

\footnotetext{
${ }^{13}$ Some experiments were run multiple times, which is why there are more dots than there are frequencies available.
} 


\begin{tabular}{|c|c|c|c|c|c|c|c|}
\hline \multirow[t]{2}{*}{ RX node } & \multirow{2}{*}{$\begin{array}{l}\text { O-QPSK } \\
\text { (250 kbps) } \\
127 \text { bytes }\end{array}$} & \multicolumn{2}{|c|}{$\begin{array}{c}\text { OFDM1@400 } \\
\text { (400 kbps) }\end{array}$} & \multicolumn{2}{|c|}{$\begin{array}{c}\text { OFDM1@800 } \\
\text { (800 kbps) }\end{array}$} & \multicolumn{2}{|c|}{$\begin{array}{c}\text { OFDM2@800 } \\
\text { (800 kbps) }\end{array}$} \\
\hline & & 127 bytes & 2047 bytes & 127 bytes & 2047 bytes & 127 bytes & 2047 bytes \\
\hline $1 \mathrm{~A}$ & $96 \%$ & $100 \%$ & $100 \%$ & $100 \%$ & $100 \%$ & $100 \%$ & $92 \%$ \\
\hline $1 \mathrm{~B}$ & $78 \%$ & $100 \%$ & $100 \%$ & $100 \%$ & $100 \%$ & $97 \%$ & $92 \%$ \\
\hline $1 \mathrm{C}$ & $91 \%$ & $100 \%$ & $100 \%$ & $100 \%$ & $100 \%$ & $100 \%$ & $100 \%$ \\
\hline 1D & $88 \%$ & $100 \%$ & $100 \%$ & $100 \%$ & $100 \%$ & $100 \%$ & $100 \%$ \\
\hline $1 \mathrm{E}$ & $81 \%$ & $100 \%$ & $100 \%$ & $100 \%$ & $100 \%$ & $100 \%$ & $100 \%$ \\
\hline $1 \mathrm{~F}$ & $62 \%$ & $94 \%$ & $92 \%$ & $89 \%$ & $89 \%$ & $85 \%$ & $75 \%$ \\
\hline $1 \mathrm{G}$ & $81 \%$ & $100 \%$ & $100 \%$ & $100 \%$ & $98 \%$ & $99 \%$ & $96 \%$ \\
\hline $1 \mathrm{H}$ & $71 \%$ & $100 \%$ & $100 \%$ & $99 \%$ & $99 \%$ & $99 \%$ & $94 \%$ \\
\hline $2 \mathrm{~A}$ & $0 \%$ & $100 \%$ & $100 \%$ & $100 \%$ & $100 \%$ & $100 \%$ & $98 \%$ \\
\hline $2 \mathrm{~B}$ & $0 \%$ & $88 \%$ & $80 \%$ & $74 \%$ & $44 \%$ & $4 \%$ & $0 \%$ \\
\hline $2 \mathrm{C}$ & $0 \%$ & $0 \%$ & $0 \%$ & $0 \%$ & $0 \%$ & $0 \%$ & $0 \%$ \\
\hline
\end{tabular}

TABLE IV: The Packet Delivery Ratio (PDR) of the wireless link for multiple positions of the RX node, for each radio setting and frame length.

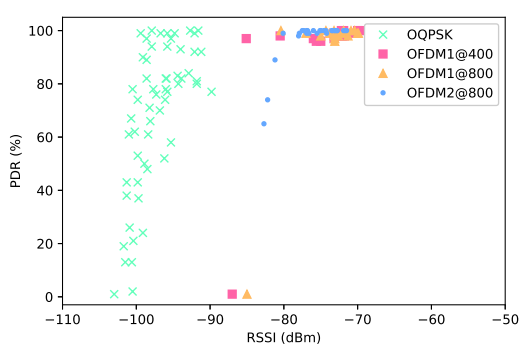

(a) node $1 \mathrm{~F}$

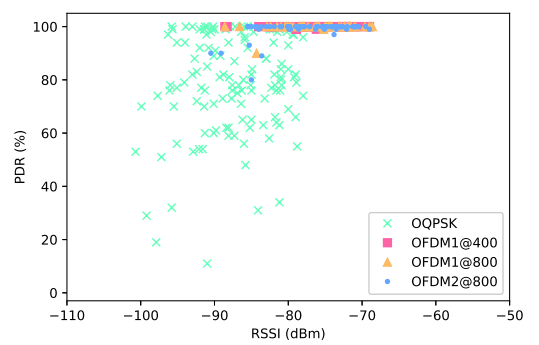

(b) node $1 \mathrm{G}$

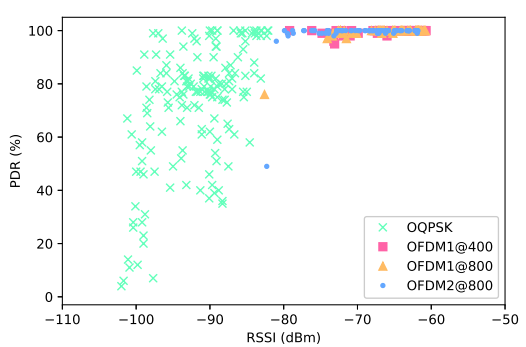

(c) node $1 \mathrm{H}$

Fig. 4: PDR vs RSSI (127 B frames).

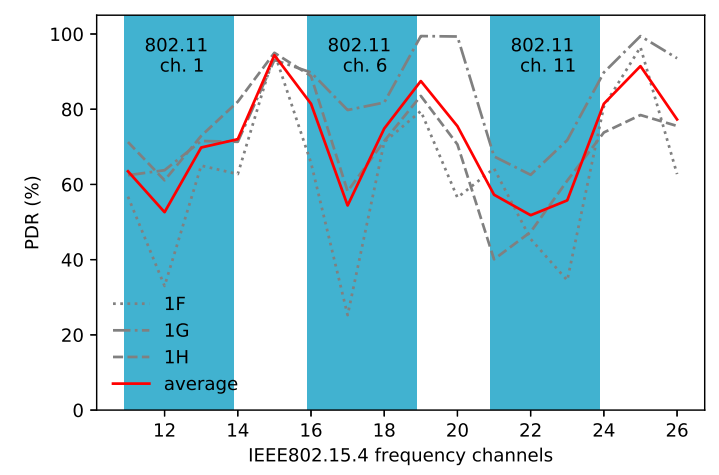

Fig. 5: Average PDR per channel on locations $1 \mathrm{~F}, 1 \mathrm{G}$ and $1 \mathrm{H}$.

The resulting recommendation is hence to use frequency repetition when the PDR of the link is marginal.

\section{The Importance of Using a Wide OFDM Band}

In order to achieve a high data rate, the radio can be configured to use a lower OFDM option (more sub-carriers in the channel) and/or a higher MCS value (higher data rate per sub-carrier). We want to explore which approach is better, from a PDR point of view. In particular, we compare OFDM1@800 and OFDM2@800: they both result in the same data rate (800 kbps), OFDM1@800 by using more sub- carriers, OFDM2@800 by increasing the data rate of each subcarrier (16-QAM has a constellation size of 16, QPSK a constellation of 4 ).

Location 2B in Table IV satisfies our trade-off. From a PDR perspective, using a wide band yields good connectivity (74\% PDR with $127 \mathrm{~B}$ ), while using a higher MCS number as in OFDM2@800 causes the communication to be almost impossible (4\% PDR with $127 \mathrm{~B}$ ).

The resulting recommendation is hence to use the lowest possible OFDM option (wider channels), even if this means fewer channels.

\section{E. Resulting Battery Lifetime Comparison}

Table III indicates the current draw of the radio in each mode $^{14}$. We assume a state-of-the-art MAC protocol, such as $\mathrm{TSCH}$, which ensures that a node's radio is only on when needed (no idle listening).

We want to compute the charge the TX node needs to successfully send a $127 \mathrm{~B}$ to an RX node, for several locations. The term "successfully" implies retransmissions: if the PDR of the link is $50 \%$, the TX node will have to transmit on average twice. Eq. (1) expresses that charge. $C$ is the charge in Coulomb, $d$ is the duration a radio needs to send the $127 \mathrm{~B}$

\footnotetext{
${ }^{14}$ There are radios on the market which draw significantly less current for O-QPSK (9.7/4.5 mA TX/RX current for Analog Devices' LTC5800). While exact numbers presented in this section will be different with different hardware, the conclusions hold.
} 


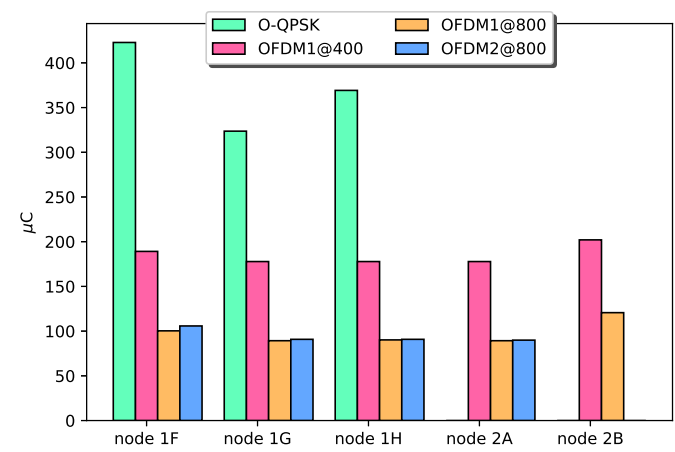

Fig. 6: Charge needed by the TX node to successfully transmit a 127-byte frame to a particular RX node, including retransmissions. For positions $2 \mathrm{~A}$ and $2 \mathrm{~B}$, the computation cannot be done for O-QPSK and OFDM2@800, as the PDR is zero.

frame, $I$ is the current the radio draws when transmitting, and PDR is the Packet Delivery Ratio (a number between 0.0 and 1.0) of the link between the transmitter and receiver nodes.

$$
C=\frac{d \times I}{P D R}
$$

Fig. 6 plots (1) for nodes $1 \mathrm{~F}-2 \mathrm{~B}$, for all 4 radio settings. OFDM is more efficient than O-QPSK, in all cases. This is because the PDR of O-QPSK is lower than OFDM, and because OFDM has a higher data rate. While a more complete benchmarking/analysis (possibly using different radios, and taking radio wake-up times and acknowledgment overhead into account), the superiority of OFDM Fig. 6 shows is so clear that the trend will still hold.

\section{CONClusions}

The overall lesson learnt from this paper is that OFDM should no longer be overlooked for low-power wireless networks, in particular in Smart Building applications. Not only are OFDM-capable radios readily available on the market, their performance meets the expectations. Their range is better than traditional $2.4 \mathrm{GHz}$ O-QPSK, with techniques such as frequency repetition very efficiently handling multi-path fading and external interference directly at the physical layer. Current OFDM radios still consume in the order of $6 \times$ more than the best-in-class $2.4 \mathrm{GHz}$ O-QPSK counterparts, but that is bound to change as inter-vendor competition kicks in.

For Smart Building applications, this paper makes the recommendation of using OFDM option 1, with MCS2 with short ( $<128 \mathrm{~B})$ frames, MCS3 otherwise.

It is clear that a MAC-layer scheme will need to be introduced (for example through the 6TiSCH standardization action) which exploits the agility of these radios: for each frame, agree with your neighbor on the most appropriate radio setting.

\section{ACKNOWLEDGMENTS}

This work is partially supported by the European Commission through the H2020 F-Interop and H2020 ARMOUR projects, and by the Spanish Ministry of Economy and the
FEDER regional development fund through the SINERGIA project (TEC2015-71303-R).

\section{REFERENCES}

[1] K. Pister, P. Thubert, S. Dwars, and T. Phinney, Industrial Routing Requirements in Low-Power and Lossy Networks, IETF Std. RFC5673, October 2009.

[2] A. Brandt, J. Buron, and G. Porcu, Home Automation Routing Requirements in Low-Power and Lossy Networks, IETF Std. RFC5826, April 2010.

[3] M. Dohler, T. Watteyne, T. Winter, and D. Barthel, Routing Requirements for Urban Low-Power and Lossy Networks, IETF Std. RFC5548, May 2009.

[4] J. Martocci, P. De Mil, N. Riou, and W. Vermeylen, Building Automation Routing Requirements in Low-Power and Lossy Networks, IETF Std. RFC5867, June 2010.

[5] IEEE Standard for Information Technology - Telecommunications and Information Exchange Between Systems - Local and Metropolitan Area Networks Specific Requirements Part 15.4: Wireless Medium Access Control (MAC) and Physical Layer (PHY) Specifications for Low-Rate Wireless Personal Area Networks (LR-WPANs), IEEE Std., 2003.

[6] T. Winter, P. Thubert, A. Brandt, J. Hui, R. Kelsey, P. Levis, K. Pister, R. Struik, J. Vasseur, and R. Alexander, RPL: IPv6 Routing Protocol for Low-Power and Lossy Networks, IETF Std. RFC6550, March 2012.

[7] H. Khaleel, C. Pastrone, F. Penna, M. A. Spirito, and R. Garello, "Impact of Wi-Fi Traffic on the IEEE 802.15.4 Channels Occupation in Indoor Environments,' in Conference on Electromagnetics in Advanced Applications, September 2009, pp. 1042-1045.

[8] T. Watteyne, C. Adjih, and X. Vilajosana, "Lessons Learned from Large-scale Dense IEEE802.15.4 Connectivity Traces," in Conference on Automation Science and Engineering, August 2015, pp. 145-150.

[9] C. Adjih, E. Baccelli, E. Fleury, G. Harter, N. Mitton, T. Noel, R. Pissard-Gibollet, F. Saint-Marcel, G. Schreiner, J. Vandaele, and T. Watteyne, "FIT IoT-LAB: A Large Scale Open Experimental IoT Testbed," in World Forum on Internet of Things (WF-IoT), December 2015, pp. 459-464.

[10] T. Watteyne, S. Lanzisera, A. Mehta, and K. S. J. Pister, "Mitigating Multipath Fading through Channel Hopping in Wireless Sensor Networks," in IEEE International Conference on Communications, May 2010, pp. 1-5.

[11] T. Watteyne, J. Weiss, L. Doherty, and J. Simon, "Industrial IEEE802.15.4e Networks: Performance and Trade-offs," in Conference on Communications (ICC). IEEE, June 2015, pp. 604-609.

[12] T. Watteyne, V. Handziski, X. Vilajosana, S. Duquennoy, O. Hahm, E. Baccelli, and A. Wolisz, "Industrial Wireless IP-Based Cyber Physical Systems," Proceedings of the IEEE, vol. 104, no. 5, pp. 10251038, May 2016.

[13] IEEE Standard for Local and metropolitan area networks-Part 15.4: Low-Rate Wireless Personal Area Networks (LR-WPANs) Amendment 3: Physical Layer (PHY) Specifications for Low-Data-Rate, Wireless, Smart Metering Utility Networks, IEEE Std., April 2012.

[14] 802.15.4-2015 - IEEE Standard for Low-Rate Wireless Networks, IEEE Std., April 2016.

[15] J. Dias, F. Ribeiro, M. Campos, R.and Ricardo, L. Martins, F. Gomes, and A. Carrapatoso, "Evaluation of an RPL/6LoWPAN/IEEE 802.15.4g Solution for Smart Metering in an Industrial Environment," in Conference on Wireless On-demand Network Systems and Services (WONS), January 2016, pp. 1-4.

[16] K. Mochizuki, K. Obata, K. Mizutani, and H. Harada, "Development and Field Experiment of Wide Area Wi-SUN System Based on IEEE 802.15.4g," in World Forum on Internet of Things (WF-IoT), December 2016, pp. 76-81.

[17] D. Hanes, G. Salgueiro, P. Grossetete, R. Barton, and J. Henry, IoT Fundamentals. Cisco Press, 2017.

[18] S. Lee, B. Kim, M. K. Oh, Y. Jeon, and S. Choi, "Implementation of IEEE 802.15.4g Wireless Communication Platform for Smart Utility Service," in Conference on Consumer Electronics (ICCE), Berlin, September 2013, pp. 287-289.

[19] ITU-R, "Recommendation ITU-R P.1238-9. Propagation Data and Prediction Methods for the Planning of Indoor Radiocommunication Systems and Radio Local Area Networks in the Frequency Range $300 \mathrm{MHz}$ to $100 \mathrm{GHz}$,' International Telecommunication Union, Tech. Rep., 062017. 